\title{
Image-Based 3D Planning of Maxillofacial Distraction Procedures Including Soft Tissue Implications
}

\author{
Filip Schutyser ${ }^{1}$, Johan Van Cleynenbreugel ${ }^{1}$, Matthieu Ferrant ${ }^{2}$, \\ Joseph Schoenaers ${ }^{3}$, and Paul Suetens ${ }^{1}$ \\ 1 Medical Image Computing (Radiology - ESAT/PSI), Faculties of Medicine and \\ Engineering, University Hospital Gasthuisberg, Herestraat 49, B-3000 Leuven, \\ Belgium \\ 2 Telecommunications Laboratory, Université Catholique de Louvain, Belgium \\ 3 Departement of Oral and Maxillofacial Surgery, University Hospitals of Leuven, \\ Herestraat 49, B-3000 Leuven, Belgium \\ Filip.Schutyser@uz.kuleuven.ac.be
}

\begin{abstract}
Osteodistraction, a technique for new bone formation by the gradual separation of bony fragments, is a possible treatment for dysplasia of the maxillofacial skeleton. However, preoperative assessment of the optimal distraction direction, of the osteotomy trajectory, and of the influence of this procedure on the outlook of a patient, is difficult to assess preoperatively.

We report on an 3D image-based planning system for osteodistraction. Basically we adhere to a scene-based approach in which image derived visualizations and additional 3D structures are co-presented and manipulated. Osteotomy simulation with user-defined cutting trajectories, virtual distraction employing biomechanical models specific to the distraction type and evaluation tools based on a cephalometric reference frame, are available in our planning environment. According to the surgeon's findings, different choices can be redone in order to optimize the therapy. To account for soft tissue implications, skin tissue deformations are calculated using a finite element elastic model based on tetrahedral elements.

We report on results in the field of unilateral mandibular distraction. A detailed overview of the planning procedure is given by a case study.
\end{abstract}

Keywords: Image guided therapy, maxillofacial surgery simulation, osteotomy, soft tissue modelling

\section{Introduction}

Osteodistraction is a possible therapy for dysplasia of the maxillofacial skeleton. Although this technique has an expanding role, a number of key issues remain difficult. 
Some of these issues are related to surgery actions (selection of an optimal distraction direction, definition of corresponding osteotomy paths), others to surgery outcome (prediction of facial outlook). Preoperative volumetric image data can be employed for better assessment of these questions, provided they are manipulated in an appropriate planning environment.

In this paper, an image-based planning system for maxillofacial distraction is presented. This preoperative planning environment includes three major components: osteotomy tools, distraction simulation and soft tissue deformation prediction.

Section 2 gives some background information about distraction osteogenesis and discusses current planning procedures and related research work. In section 3, our developments concerning osteotomy, distraction simulation and prediction of soft tissue deformation are explained. In section 4, examples are given of the current use of the planning system for unilateral mandibular distraction. Concluding remarks finish the paper in section 5 .

\section{Background}

Distraction osteogenesis is a technique for new bone formation by the gradual separation of bony fragments. The method, although initially developed for limb lengthening, is now being applied in the treatment of craniofacial deformities. The technique offers considerable advantages over previous methods such as osteotomy and inlay bone grafting. Donor site morbidity is avoided, the investing soft tissue envelope is concurrently expanded, and the magnitude of the procedure is less. However, the technique is still in its infancy and requires further modification and refinement before it will be widespread accepted as a treatment in mainstream craniofacial surgery. Distraction osteogenesis is of increasing importance in craniofacial surgery [1].

Two major groups of craniofacial distractrion procedures are distuingished. Mandibular osteodistraction is applied for congenital deformities such as hemifacial microsomia (unilateral lengthening) and Nager's syndrome (bilateral lengthening) [2]. On the other hand, maxillar osteodistraction includes rapid maxillary expansion [34] as well as midface advancement [5].

The planning of distraction procedures currently involves evaluation of panoramic and cephalometric X rays, photographs and articulator mounted dental casts, as well as a clinical examination. Based on various measurements and using cephalometric standards, formulas can be used to compute a distraction direction [6].

Most of the cases suitable for distraction osteogenesis are problems with an important 3D effect that cannot be planned precisely with only 2D cephalometry. Furthermore, without 3D preoperative surgery planning, the direction and maximum length of distraction can only be determined during the operation itself. One should keep in mind that the amount of distraction is the sole parameter that can postoperatively be modified. Therefore, $3 \mathrm{D}$ preoperative planning holds a large potential. 
An early attempt in craniofacial planning systems is described by Cutting et al. [7]. Based on image derived 3D landmarks, bone fragments are automatically repositioned according the Bolton normative standards. More recently, they report on optical tracking systems for the positioning of bone fragments in multisegment craniofacial surgical procedures 8 .

Santler et al. 9] discuss preoperative planning using materialized models. The surgery is simulated on these models. The results are satisfactory, but disadvantages are the high extra cost and the production time for large models, and the limitation to do only one simulation per model. Moreover, all information about soft tissues is lost.

Because of the high impact of distraction therapy on the patients face, prediction of the deformation of soft tissues is highly desirable. Therefore, our work also includes a soft tissue model of the skin (i.e. the dermis and the underlying structures like fat and muscles).

Last year, we described a voxel-based approach for soft tissue modelling [14]. Although voxel-based modelling is advantageous from an image processing point of view (data remain available on a (subsampled) voxel grid), it is hard to associate biomechanical properties with the parameters of this model. Therefore, we switched our attention to models with a better biomechanical fondation.

Fung [10] reports on the biomechanical properties of living tissues. Skin tissues are called quasi-linear viscoelastic materials, meaning that these tissues show creep, relaxation and hysteresis when applying large oscillations around equilibrium, but the characteristics can be well approximated with linear viscoelasticity applying small oscillations. However, this modelling implies demanding computations which are in this application area unrealistic.

Different approaches have been investigated to reduce the computational cost. Teschner et al. describe a multilayer spring model [11, resulting in short simulation times, however no validation study is plublished. Koch et al. [12] model skin tissue as incompressible elastic tissue. They use prisms as finite elements. However, as future work, they suggest to move towards tetrahedral elements giving more topological freedom. In both cases, the meshing step, based on the approach of Waters [13, is rather tedious and error-prone.

In this paper, an isotropic homogenous elastic model for the soft tissues of the skin is presented. As a solution method, a finite element method using tetrahedral elements is chosen.

\section{Methods}

\subsection{D Simulation Environment for Maxillofacial Distraction}

Our simulation environment is based on the ideas we have previously described in [14. Basically we adhere to a scene-based approach in which image derived visualizations (MPR, surfaces, volume renderings) and additional 3D structures (external to the medical image volume) are co-presented and manipulated. 
From CT images, bone structures and the skin surface are segmented by thresholding. To provide the patient with a realistic view of the natural complexion of his/her face, we also acquire a 3D photograph. Currently we use an "active" 3D system (projecting a pattern on the face and recovering 3D from the deformation of the pattern on the $2 \mathrm{D}$ picture), see [15]. The surface thus obtained is registered with the skin surface extracted from the CT using point/surface matching algorithms.

A first step in planning osteodistraction, is osteotomy simulation. The surgeon draws a trajectory on the bone surface and adds a cutting direction and depth. With this cutting blade, a virtual cutting action is performed.

Next, the distractor is planned by indicating its fixation points on the osteotomized bone parts. The orientation of the distraction line is a crucial parameter. In a virtual distraction, movements of the bone fragments are simulated using a biomechanical model specific for the distraction type.

The result is evaluated based on a cephalometric reference frame. In this simulation environment, the surgeon can decide to redo some of his previous choices, like the distraction direction or the definition of the osteotomy blade. Thanks to a menu-based approach piloting a user through the planning procedure, user-friendliness and time-efficiency are important features of this system.

\subsection{Soft Tissue Modelling}

The soft tissues are assumed to behave as an isotropic linear elastic continuum. So, the constitutive equation can be written as equation (1), with $\epsilon_{x x}, \epsilon_{y y}, \epsilon_{z z}$, $\gamma_{x y}, \gamma_{y z}, \gamma_{z x}$ strain components, $\lambda$ en $\mu$ the Lamé constants which are a function of the Young modulus $(E)$ and the Poisson's ratio $(\nu): \lambda=\frac{E \nu}{(1+\nu)(1-2 \nu)}$ en $\mu=$ $G=\frac{E}{2(1+\nu)}$. Typical values are $E \in[2000,4000] \mathrm{Pa}$ and $\nu \in[0.25,0.4]$.

$$
\left(\begin{array}{c}
\sigma_{x x} \\
\sigma_{y y} \\
\sigma_{z z} \\
\tau_{x y} \\
\tau_{y z} \\
\tau_{z x}
\end{array}\right)=\left(\begin{array}{cccccc}
\lambda+2 \mu & \lambda & \lambda & 0 & 0 & 0 \\
\lambda & \lambda+2 \mu & \lambda & 0 & 0 & 0 \\
\lambda & \lambda & \lambda+2 \mu & 0 & 0 & 0 \\
0 & 0 & 0 & 2 \mu & 0 & 0 \\
0 & 0 & 0 & 0 & 2 \mu & 0 \\
0 & 0 & 0 & 0 & 0 & 2 \mu
\end{array}\right)\left(\begin{array}{c}
\epsilon_{x x} \\
\epsilon_{y y} \\
\epsilon_{z z} \\
\gamma_{x y} \\
\gamma_{y z} \\
\gamma_{z x}
\end{array}\right)
$$

As a result of the distraction simulation, displacements of bone fragments are known. These data are translated into boundary conditions for the soft tissue model. To solve the equilibrium equations (partial differential equations) with these boundary conditions, a finite element formulation is chosen. The finite elements are tetrahedra with a linear interpolation function.

Creating a volumetric tetrahedral mesh for irregular shapes, such as human organs, is a non-trivial task. Labelling soft tissues of the skin on the medical image data using semi-automatic segmentations tools, results in a volumetric description on a rectilinear voxel grid. By dividing the volume into cubes, and further subdividing the cubes into tetrahedra using the label values at the corners of the cubes, a tetrahedral mesh is built [16]. This approach enables us to 
automatically create tetrehadral meshes with a good level of detail. Based on this information, the mesh is deformed using our parallelized implementation of the finite element method.

\section{Results: Unilateral Mandibular Distraction}

Next, we report on applying our approach in the case of unilateral mandibular distraction. This therapy is a possible treatment for jaw asymmetry in children with hemifacial microsomia. The hypoplastic side of the mandible is osteotomized and distracted (see figure 1 and 2). Difficult key issues for this procedure are the determination of a distraction direction in order to get a symmetrical face while deciding on an osteotomy trajectory that does not damage the mandibular nerve.

Constraints typical for success in unilateral mandibular distraction are incorporated in the surgical simulator. A first constraint is facial symmetry. To provide visual inspection of the asymmetry, the normal midline of the face is determined on the skin surface. In addition, we verify different distances on each side of the mandible reflecting the symmetry: distance between "incisura mandibulae - angulus mandibulae", "incisura mandibulae - protuberantia mentalis" and "angulus mandibulae - protuberantia mentalis". A second constraint concerns the degrees of freedom in the temporomandibular joint. We model this joint as a ball-and-socket joint.

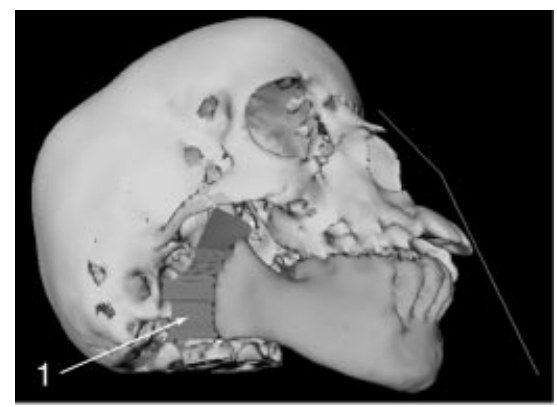

(a)

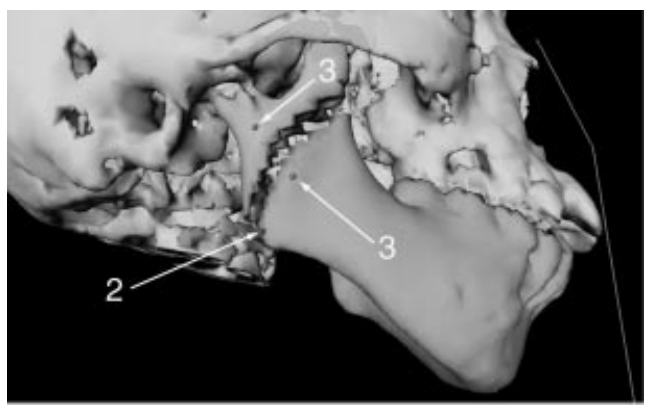

(b)

Fig. 1. This figure illustrates the planning procedure. In a first step (a) an osteotomy trajectory (1) is defined. After performing the virtual cutting action, the surgeon annotates anatomical landmarks defining important distances (2) and the distraction direction (3). Then, the surgeon can simulate and preoperatively validate the distraction. During this phase, the surgerical plan can be adapted, e.g. redefining the distraction direction and/or choosing a more suitable osteotomy trajectory. 


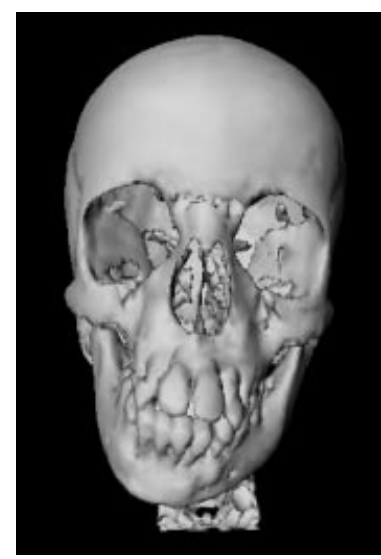

(a)

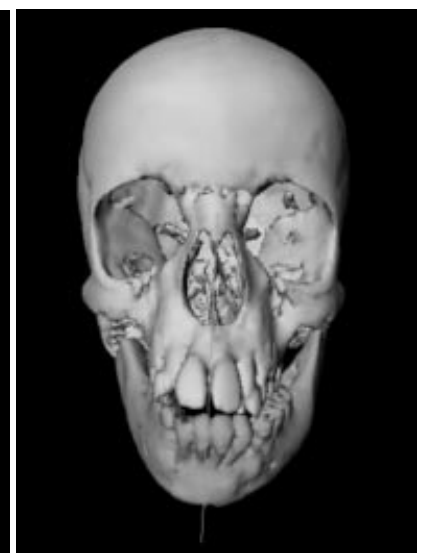

(b)

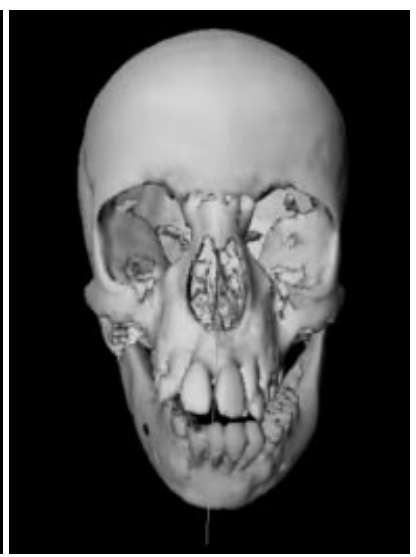

(c)

Fig. 2. After trying different possibilities for the distraction, the most suitable planning is chosen. To show the results three pictures can be compared:

(a) preoperative situation

(b) a distraction of $6 \mathrm{~mm}$ reaches a maximal symmetry.

(c) an overcorrected distraction of $9 \mathrm{~mm}$ is chosen to compensate for grow deficiencies.

After an optimal distraction direction and osteotomy trajectory have been defined and after a virtual distraction has been simulated, the soft tissue implications are calculated. Initiated by the displacements of the bone fragments, the deformation is computed using an elastic FEM model for the skin (see figure 3).

Additionally, the preoperative plan can be transferred to the operating theatre. A customized mechanical guide can be applied on the exposed jaw bone to indicate the osteotomy and the distraction direction [17.

Postoperative CT-imaging enables us to discuss the system (see figure 4). After registration of the planned and actual shape of the mandible, we noticed a good correspondance. When comparing the postoperative position of the mandible in the temporomandibular joint with the planning, the postoperative mandible translated slightly back- and upward. Finally, validating the soft tissue deformation prediction is difficult due to the skeletal differences between planning and postoperative result. Therefore, we adapted the planning, so that the match with the postoperative skull is better. After recomputing the soft tissue deformation, and computing a distance field between both surfaces, fairly small errors were found: $1 \mathrm{~mm} \pm 0.5 \mathrm{~mm}$ with peak values around the lips $(4 \mathrm{~mm})$. 


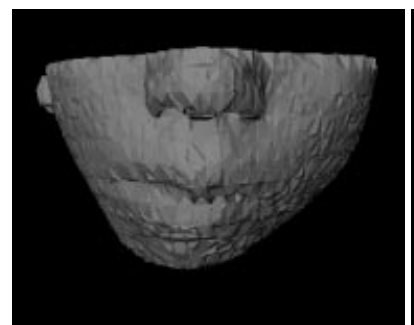

(a)

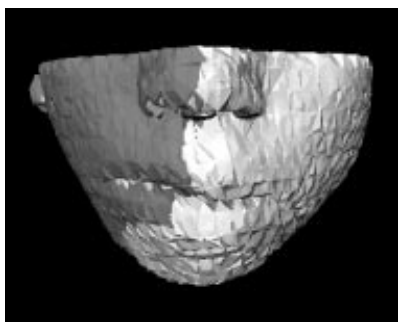

(b)

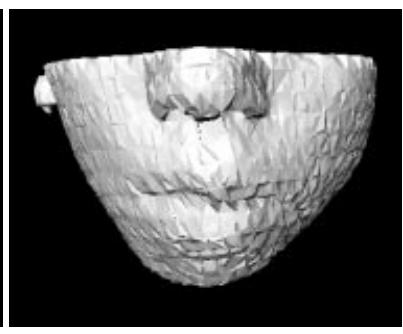

(c)

Fig. 3. This figure shows the boundary surface of the tetrahedral mesh of the soft tissues. (a) depicts the preoperative skin surface. In (b), both preoperative (dark) and expected surgical result (bright) are shown together. Figure (c) illustrates the planned result.

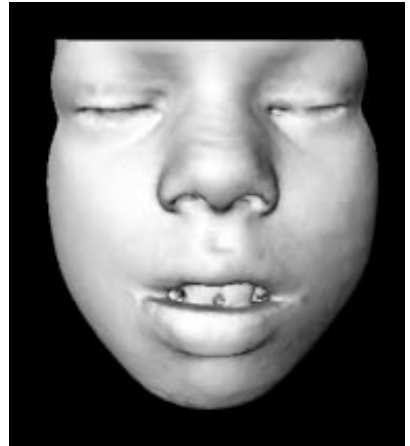

(a)

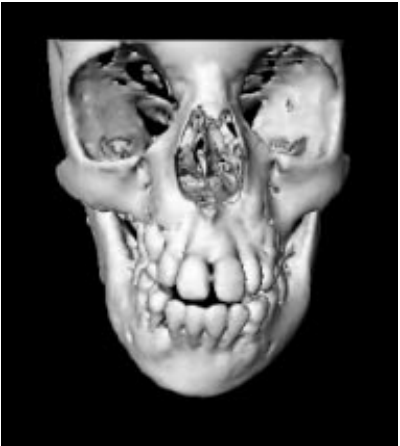

(b)

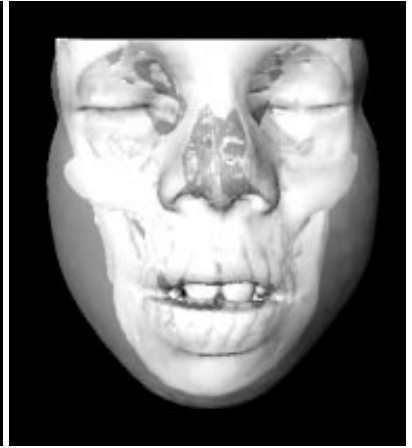

(c)

Fig. 4. From the left to the right, the postoperative skin surface, the postoperative skull and a combination of both is depicted. From postoperative CT-imaging, validation of the accuracy of our planning is possible.

\section{Conclusion}

In this paper, we presented a planning system for planning distraction and predicting deformation of soft tissue. Key issues, such as the selection of an optimal distraction direction, the definition of an osteotomy, and predicting of the influence of this procedure on the outlook of a patient, are addressed in our system. Using this preoperative planning environment, trying out and validating different distractions in 3D, without large extra costs nor losing time becomes possible.

These early results of our approach opens up alleys for future work. More clinical cases and different types of distraction are planned in the near future. 
At the time of submission, work concerning bilateral mandibular distraction, midface advancement by sagittal (forward and downward) distraction of the maxilla are investigated. Furthermore, the soft tissue modelling will be further tested and refined. The need to incorporate incompressibility, nonlinear elastic material properties and to distinguish different types of soft tissue such as fat and muscle, are to be investigated.

\section{Acknowledgments}

The work discussed here belongs, partly to the EU-funded Brite Euram III PISA project (nr. BRPR CT97 0378), a collaboration between Materialise NV, Belgium; Philips Medical Systems BV, the Netherlands: ICS-AD; DePuy International Ltd, UK; Ceka NV, Belgium; K.U. Leuven, Belgium: ESAT/Radiology Div. Biomechanics; University of Leeds, UK: Research School of Medicine, and to a grant for research specialization from the Flemish Institute for stimulation of the scientific-technological research in the industry (IWT) to Filip Schutyser.

\section{References}

1. K. Tavakoli, K.J. Steward, M.D. Poole: Distraction Osteogenesis in Craniofacial Surgery: A Review. Annals of Plastic Surgery, Vol. 40, Nr. 1, p 88-99, January 1990

2. J.G. McCarthy, J. Schreiber, N. Karp: Lengthening of the human mandible by gradual distraction. Plast. Reconstr. Surg. 1992; 89:1-8.

3. J.A. Lehman, A.J. Haas: Surgical-orthodontic correction of transverse maxillary deficiency. Clinical Plastic Surgery, Vol. 16, p 749-775, 1989

4. C. Stromberg, J. Holm: Surgically assisted, rapid maxillary expansion in adults: a retrospective long-term follow-up study. Journal of Craniomaxillofacial Surgery, Vol. 23, p 222-227, 1995

5. J.W. Polley, A. Figueroa: Rigid External Distraction: Its Application in Cleft Maxillary Deformities. Plastic and Reconstructive Surgery, Vol. 102, Nr. 5, p 1360-1374, 1998

6. H.W. Losken, G.T. Patterson, S.A. Lazarou, T. Whitney: Planning Mandibular Distraction: a priliminary report. Cleft Palate-Craniofacial Journal, Jan, 1995, vol. 32 , no. 1 , p. $71-76$

7. C. Cutting, F.L. Bookstein, B. Grayson, L. Fellingham, J.G. McCarthy: Threedimensional Computer-assisted Design of Craniofacial Surgical Procedures: Optimization and Interaction with Cephalometric and CT-based Models. Plastic and Reconstructive Surgery, vol. 77:6, p. 877-885, 1986

8. C. Cutting, B. Grayson, J.G. McCarthy, C. Thorne, D. Khorramabadi, B. Haddad, R. Taylor: A Virtual Reality System for Bone Fragment Positioning in Multisegment Craniofacial Surgical Procedures. Plastic and Reconstructive Surgery, vol. 102:7, p. 2436-2443, 1998

9. G. Santler, H. Kärcher, A. Gaggl, G. Schultes, R. Mossböck: Advantage of ThreeDimensional Models in Intraoral Callus Distraction. Computer Aided Surgery, vol. 3, no. 3, p. 99-107, 1998

10. Y.C. Fung: Biomechanics: Mechanical properties of Living Tissues. 2nd edition. Ch. 7, Springer-Verlag, 1993, p. 242-320 
11. M. Teschner, S. Girod, B. Girod: Optimization Approaches for Soft-Tissue Prediction in Craniofacial Surgery Simulation. Proceedings 2nd international conference on medical image computing and computer-assisted intervention - MICCAI'99, lecture notes in computer science, vol. 1679, p. 1183-1190, September 19-22, 1999

12. R.M. Koch, M.H. Gross, F.R. Carls, D.F. von Büren, G. Fankhauser, Y. Parish: Simulating Facial Surgery Using Finite Element Methods. SIGGRAPH 96 Conference Proceedings, 1996, p. 421-428

13. K. Waters: A physical model of facial tissue and muscle articulation derived from computer tomography data. SPIE Vol. 1808, Visualization in Biomedical Computing, volume 21, p. 574-583, 1992.

14. F. Schutyser, J. Van Cleynenbreugel, J. Schoenaers, G. Marchal, P. Suetens: A Simulation Environment for Maxillofacial Surgery Including Soft Tissue Implications. Proceedings 2nd international conference on medical image computing and computer-assisted intervention - MICCAI'99, lecture notes in computer science, vol. 1679, pp. 1210-1217, September 19-22, 1999

15. ShapeSnatcher, www.eyetronics.com

16. M. Ferrant, S.K. Warfield, C.R.G. Guttmann, R.V. Mulkren, F.A. Jolesz, R. Kikinis: 3D Image Matching Using a Finite Element Based Elastic Deformation Model. Proceedings 2nd international conference on medical image computing and computer-assisted intervention - MICCAI'99, lecture notes in computer science, vol. 1679, p. 202-209, September 19-22, 1999

17. F. Schutyser, J. Van Cleynenbreugel, N. Nadjmi, J. Schoenaers, P. Suetens: 3D image-based planning for unilateral mandibular distraction. Proceedings 14th international congress and exhibition on computer assisted radiology and surgery, $\mathrm{p}$. 899-904, CARS 2000, June 28 - July 1 2000, San Francisco, USA 